\title{
DEZ ANOS DA POLÍTICA PÚBLICA INTERSETORIAL PROGRAMA SAÚDE NA ESCOLA: PANORAMA DESCRITIVO POR ANÁLISE DOCUMENTAL
}

\author{
TEN YAERS OF THE INTERSECTORAL PUBLIC POLICY HEALTH IN \\ SCHOOL PROGRAM: DESCRIPTIVE PANORAMA BY DOCUMENTAL \\ ANALYSIS
}

\author{
Luna Marquez Ferolla* \\ Cláudia Souza Passador \\ João Luiz Passador ${ }^{* * *}$
}

\section{RESUMO}

O Programa Saúde na Escola (PSE) completou uma década de implementação, durante a qual passou por expansão e reestruturações normativas. Para entender melhor essa trajetória, este trabalho tem o objetivo de caracterizar o PSE enquanto política pública intersetorial, traçando um panorama descritivo por análise de documentos oficiais, a partir de referencial teórico que conjuga políticas públicas intersetoriais e a evolução do movimento de educação em saúde. Foram analisados 51 documentos publicados entre 2007 e 2018, contendo atos normativos, relatórios e manuais de orientação. Constatou-se que o programa mantém os aspectos essenciais da proposta inicial, como objetivos, diretrizes e distribuição de responsabilidades entre os entes federativos; mas sofreu alterações consideráveis na estrutura normativa. Foram identificadas as fases: piloto, expansão, universalização e consolidação. Percebe-se que o início do programa foi marcado por irregularidades e adaptações, mas houve um movimento de harmonização normativa no intuito de aproximar a proposta da realidade percebida.

Palavras-chave: Programa Saúde na Escola; Políticas públicas intersetoriais; Educação em saúde; Análise documental.

\begin{abstract}
The Health in School Program (HSP) has been implemented for a decade, during a journey of expansion and standards restructuring. Aiming a better understanding of this journey, this paper characterizes PSE as an intersectoral policy, outlining a descriptive panorama by document analysis, based in a theoretical framework that brings together a discussion on intersectoral policy and the evolution of the health education movement. 51 written documents published between 2007 and 2018 were analyzed, containing normative documents, reports and guidance manuals for implementers. It was possible to verify that the program maintains the most important functions of the initial agenda, as objectives, guidelines and distribution of responsibilities between federal actors; but it has undergone considerable changes in the normative structure. There are four phases: pilot, expansion, universalization and consolidation. It is noticed that the beginning of the program was marked by irregularities and adaptations, but it there is a movement of harmonization of the norms aiming to approach a proposal of perceived reality.
\end{abstract}

Key-Words: Health in School Program; Intersectoral public policies; Health education; Documentary analysis.

\footnotetext{
"Doutora em Administração de Organizações pela Faculdade de Administração, Contabilidade e Economia de Ribeirão Preto-SP (FEA-RP/USP).

"Doutora em Educação pela Universidade de São Paulo (USP).

*** Doutor em Administração de Empresas pela Fundação Getúlio Vargas (FGV-SP).
} 


\section{INTRODUÇÃO}

A escola é considerada espaço privilegiado para desenvolvimento de práticas intersetoriais, pelo fato de ser um espaço onde é possível atingir simultaneamente um grande número de indivíduos e, dado seu caráter educativo, propício para a assimilação e compreensão de informações, ter a potencialidade de promover mudança efetiva de comportamentos e hábitos (MONTEIRO; BIZZO, 2014). Tal premissa fundamenta a promoção de ações diversas, no intuito de promover conscientização em relação a temas relevantes para a sociedade, cujo impacto se dá, tanto pela formação cidadã dos próprios beneficiários diretos (crianças e adolescentes) quanto pela ação multiplicadora que eles podem ter ao atuar como agentes de mudança em suas comunidades e grupos de convivência (CUMMINGS; SCHERMERHORN, 2003; HOLLAND, 2010; MORROW, 1999; WOOD et al., 2013).

Dentre as áreas que podem se beneficiar de tais ações, destaca-se a saúde que é inerentemente intersetorial, especialmente quando considerada no âmbito coletivo, por sofrer influência de fatores que vão além da oferta e da qualidade dos serviços do setor em si, chamados determinantes sociais da saúde (DSS) (BUSS, 2000; DUBOIS; ST-PIERRE; VERAS, 2015; MARMOT etal., 2008; NDUMBE-EYOH; MOFFATT, 2013). Dentre as áreas ligadas intrinsicamente à saúde pública, a educação se destaca por seu caráter catalizador de desenvolvimento (KLIKSBERG, 2003; PIRES, 2005; PSACHAROPOULOS; PATRINOS, 2004), pelo potencial de impacto nos níveis individual e coletivo, com efeitos imediatos e duradouros e pela profusão de relatos de casos de sucesso, especialmente acerca de ações desenvolvidas no ambiente escolar (AZEVEDO; PELICIONI; WESTPHAL, 2012; DAHLGREN; WHITEHEAD, 1991; GARCIA et al., 2014; NDUMBE-EYOH; MOFFATT, 2013; POTVIN, 2012).

Contemplando a relação mencionada, foi lançado em 2007 o Programa Saúde na Escola ${ }^{1}$, política pública federal intersetorial, desenvolvida em conjunto pelos Ministérios da Saúde (MS) e da Educação (MEC), no intuito de fomentar e subsidiar ações de promoção, prevenção e atenção à saúde o ambiente escolar. O programa prevê implementação no âmbito municipal, pelas Equipes de Saúde da Família (EqSF) em escolas da rede pública de educação básica. A adesão é voluntária, com repasse de recursos financeiros aos que aderem. Há uma lista de sugestões para a atuação dos municípios, incluindo ações de avaliação clínica e psicossocial dos estudantes, com acompanhamento de seu estado de saúde, promoção e prevenção, formação de profissionais e jovens para atuarem como multiplicadores e o monitoramento do próprio programa (MINISTÉRIO DA SAÚDE, 2007).

O programa completou uma década de implementação, durante a qual passou por uma fase piloto, seguida de expansão e reestruturações normativas. Para entender melhor essa trajetória, o presente trabalho foi proposto com o objetivo de caracterizar o PSE enquanto política pública intersetorial, traçando um panorama descritivo a partir de análise de documentos oficiais.

\section{POLÍTICAS PÚBLICAS INTERSETORIAIS PARA SAÚDE}

No Brasil a ideia da intersetorialidade aparece na década de 70, no II Plano Nacional de Desenvolvimento Econômico (1975), tendo sido mencionada também em programas específicos relacionados à saúde, saneamento e nutrição (FERREIRA; SILVA, 2005). A criação do SUS impulsiona o ideário ao preconizar a "integração em nível executivo das ações de saúde, meio ambiente e saneamento básico"2. Nesse entremeio a aprovação da Política Nacional de Promoção da Saúde (PNPS) ${ }^{3}$ incorpora de forma mais íntegra e acurada o conceito de intersetorialidade, reconhecendo sua indispensabilidade ao atestar: "a impossibilidade do setor sanitário responder sozinho à transformação dos determinantes e condicionantes para garantir opções saudáveis para a população" (MINISTÉRIO DA SAÚDE, 2006, p. 10). O documento define intersetorialidade como "uma articulação das possibilidades dos distintos setores de pensar a questão complexa da saúde, de corresponsabilizar-se pela garantia da saúde como direito humano e de cidadania, e de mobilizar-se na formulação de

\footnotetext{
${ }^{2}$ Lei $\mathrm{n}^{\text {a }} 8.080$, de 19 de setembro de 1990 , artigo $7^{\circ}$, inciso $\mathrm{X}$

${ }^{3}$ Portaria no 687, de 20 de março de 2006.
}

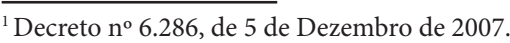


intervenções que a propiciem" (MINISTÉRIO DA SAÚDE, 2006, p. 10).

O conceito reverbera como um novo paradigma da Saúde Pública ao abranger as diversas causas do binômio saúde-doença, partindo de valores como democratização, estímulo à participação social, equidade e promoção da sustentabilidade. O ideário conduz à percepção da saúde como produto de um amplo espectro de fatores (físico, econômico, social, cultural, ambiental e político), em um complexo processo que demanda articulação de saberes técnicos e populares, mobilização de recursos institucionais e comunitários, na proposição de soluções intersetoriais (AZEVEDO; PELICIONI; WESTPHAL, 2012; PELICONI, 2005).

É papel do Estado na promoção da equidade em saúde assumir a responsabilidade de desenvolver sistemas flexíveis, que facilitem o acesso e a participação, fomentando as relações cooperativas entre cidadãos e instituições. Ao tecer considerações sobre a ação política, discute-se três direções estratégicas fundamentais, com ênfase particular no combate às iniquidades em saúde: 1) abordar o contexto; 2) ação intersetorial; e 3) participação social e empoderamento, evidenciando o protagonismo do governo como promotor de políticas públicas intersetoriais para promoção da saúde (SOLAR; IRWIN, 2010).

Nesse contexto, entende-se 'política pública' como um conjunto de diretrizes intencionalmente elaboradas por um grupo de atores, com centralidade do Estado, para mitigar um problema público, como reflexo de conteúdos concretos e simbólicos de decisões políticas (JENKINS, 1978; PARSONS, 1995). A análise de políticas públicas usualmente remete à um processo cíclico dividido nas seguintes etapas sequenciais, mas interdependentes: formação de agenda; formulação de alternativas; tomada de decisão, implementação; avaliação (FREY, 1996; HOWLETT; RAMESH; PERL, 2013; SECCHI, 2010).

Dada a multiplicidade de atores envolvidos, é válido mencionar o conceito de "políticas integradas", sobre o qual (OLIVEIRA, 2019, p. 80) explica: "a integração horizontal se refere às ações de intersetorialidade das políticas, e a vertical se destina a compreender as ações entre os níveis de governo", sugerindo que as políticas públicas sociais federais, como é o caso do PSE, devem englobar ambas as dimensões. A intersetorialidade é então complementar à integração, agregando esforços dos diversos setores que podem atender às demandas de sujeitos e cenários complexos, multicausais e relacionados entre si (OLIVEIRA, 2019), como a saúde e a educação.

A condução de políticas públicas intersetoriais demanda que sejam conjugados os componentes de objetivos e processos, que alinhados proporcionam ações consideradas consistentes e sinérgicas. Destarte, podem ser elaboradas ferramentas de gestão e avaliação das ações conduzidas, contribuindo com a responsabilização dos atores envolvidos e o aumento da transparência (POLLITT, 2003; WU et al., 2014). Para que o modelo de políticas públicas sociais integrado seja efetivo "são imprescindíveis instrumentos e mecanismos de coordenação, capacidade de construção de agendas e coalizações que ultrapassem as questões políticas, técnicas e de gestão" (OLIVEIRA, 2019, p. 82).

Convém mencionar que para lidar com a complexidade dos cenários multidimensionais nos quais se desenvolve, a gestão da intersetorialidade nas políticas públicas demanda integração entre as práticas de planejamento, implementação, monitoramento e avaliação, de forma a permitir tanto o adequado acompanhamento dos atores envolvidos nestas etapas quanto o controle social por qualquer outro interessado (CARMO; GUIZARDI, 2017; INOJOSA, 1998; OLIVEIRA, 2019).

\section{EDUCAÇÃO EM SAÚDE}

Temas ligados à saúde têm sido tradicionalmente abordados nas escolas brasileiras, mas de formas bastante distintas, em um movimento que se inicia no século XIX e passa por várias configurações, até chegar no formato atual. Ao recapitular a história da saúde na escola no Brasil (CASEMIRO; FONSECA; SECCO, 2014; SILVA; BODSTEIN, 2016), são reconhecidos cinco modelos (Quadro 6), consoantes com a evolução do sistema de saúde em si, que por sua vez reflete os conceitos de saúde em voga nos momentos correspondentes (SANTOS; WESTPHAL, 1999). 
FIGURA 1 - Linha do tempo: modelos orientadores de ações de saúde na escola

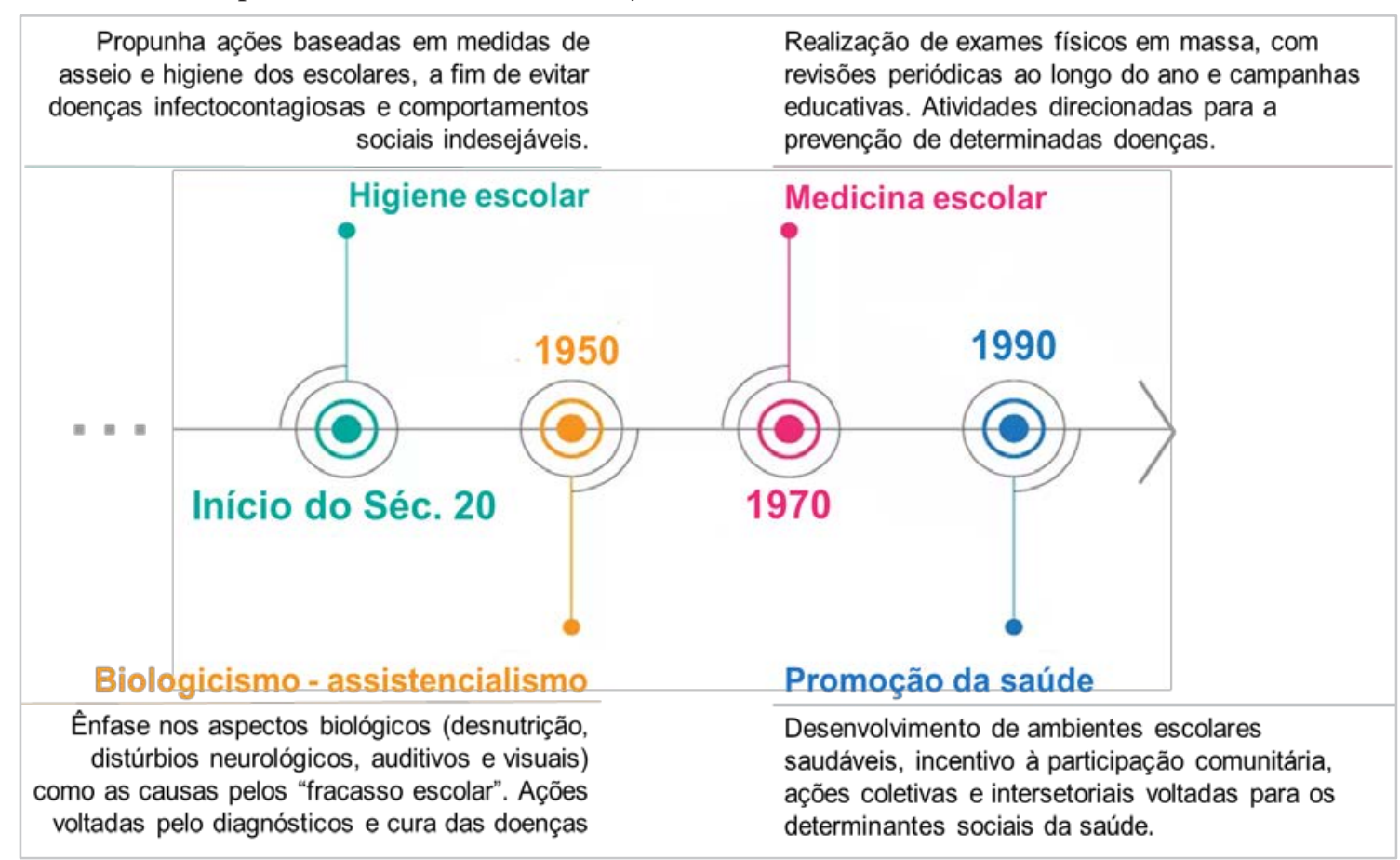

Fonte: Adaptada de ANDRADE, D. A. Intersetorialidade no Programa Saúde na Escola: uma análise sobre a construção de redes entre saúde e educação, no município do Recife. Mestrado em Saúde Pública-Recife: Fundação Oswaldo Cruz, 2015.

O primeiro momento, chamado de modelo higienista, predomina do final do século XIX até meados do século XX. A partir de uma visão reducionista da saúde focada em evitar doenças e agravos, nas escolas a preocupação central são as condições sanitárias e as ações giram em torno de higiene e alimentação, preconizando modelos comportamentais a serem seguidos (CASEMIRO; FONSECA; SECCO, 2014; COUTO et al., 2016; FIGUEIREDO; FIGUEIREDO, 1986; MONTEIRO; BIZZO, 2014).

O segundo modelo, denominado assistencialista ou biomédico, ganha corpo entre as décadas de 1950 e 1960 e vai até os anos 1990. A visão sobre o conceito de saúde é ainda limitada, com valorização das especializações e da hospitalização. Nas escolas, o estudante passa a ser avaliado como um todo, embora em uma perspectiva biomédica. Ainda que sejam incluídos no currículo temas transversais, o professor é o agente transmissor de informações e a criança mero receptor passivo (CASEMIRO; FONSECA; SECCO, 2014; COUTO et al., 2016; MONTEIRO; BIZZO, 2014).

O terceiro momento, que se inicia em meados da década de 70, é focado no monitoramento do estado de saúde dos estudantes, com realização de avaliação clínica e campanhas de conscientização direcionadas para a prevenção de doenças específicas consideradas alarmantes em cada contexto. Segue a visão de saúde voltada para a hospitalização e a especialização (Andrade, 2015).

O quarto modelo foi impulsionado pela Iniciativa Regional Escolas Promotoras de Saúde (IREPS), lançada pela a Organização Pan-Americana de Saúde (OPAS) em 1995 e representou considerável avanço. Passa-se a valorizar a promoção da saúde como bem-estar social, considerando a relevância do coletivo, o que demandou o estabelecimento de ações integradas entre as áreas de educação e saúde. Nesse sentido, governos federal, estaduais e municipais passam a promover iniciativas de educação em saúde em ambiente escolar, dentre as quais se destaca o Projeto Saúde e Prevenção nas Escolas (SPE), lançado em 2003 e incorporado posteriormente pelo PSE (CASEMIRO; FONSECA; SECCO, 2014; COUTO et al., 2016; MONTEIRO; BIZZO, 2014).

O quinto momento, ainda vigente, pode ser considerado um desdobramento do anterior, pois conserva grande parte das suas características. No entanto, traz avanços no sentido de intensificar a cooperação intersetorial, indo além da educação e da saúde, 
envolvendo outras áreas, nos três níveis federativos, além da corresponsabilização das famílias e comunidade. A visão norteadora é a da saúde como estado de completo bem-estar físico, mental e social, o que leva à valorização das ações de promoção e prevenção e da atenção básica (CASEMIRO; FONSECA; SECCO, 2014; COUTO et al., 2016; FARIAS, 2014; MONTEIRO; BIZZO, 2014; VIEIRA, 2013).

É nesse contexto que o governo federal lança, em 2006, do Programa Saúde na Escola (PSE) ${ }^{1}$, política pública federal intersetorial, desenvolvida em conjunto pelos Ministérios da Saúde (MS) e da Educação (MEC), no intuito de fomentar e subsidiar ações de promoção, prevenção e atenção à saúde na rede pública de educação básica.

\section{ASPECTOS METODOLÓGICOS}

Consulta ao Fundo Nacional de Saúde (FNS) ${ }^{2}$ mostrou que foi repassado aos municípios, entre 2011 e 2017, com dotação ao PSE, um total de quase 310 milhões de reais, com os seguintes montantes a cada ano (TABELA 1):

Tabela 1 - Total dos recursos financeiros repassados aos municípios

\begin{tabular}{l|l}
\hline Ano & Total repassado - PSE \\
\hline 2011 & $\mathrm{R} \$ 46.929 .855,00$ \\
\hline 2012 & $\mathrm{R} \$ 66.537 .835,00$ \\
\hline 2013 & $\mathrm{R} \$ 58.508 .319,00$ \\
\hline 2014 & $\mathrm{R} \$ 2.665 .683,00$ \\
\hline 2015 & $\mathrm{R} \$ 66.830 .206,00$ \\
\hline 2016 & $\mathrm{R} \$ 8.460 .112,00$ \\
\hline 2017 & $\mathrm{R} \$ 58.966 .246,00$ \\
\hline Total & R\$ 308.898.256,00 \\
\hline
\end{tabular}

Fonte: Consulta ao Fundo Nacional de Saúde, em dezembro de 2018.

Percebe-se que é alta a variação dos valores entre os anos. Desde o início da implementação, no ano letivo de 2009, o programa já está na sua terceira configuração, com modificações em aspectos como: triagem de municípios aptos, processos de adesão e monitoramento, critérios e valores para repasse de recursos, ações a serem desenvolvidas nas escolas. Em busca de compreender com maior clareza os diversos arranjos, foi realizada análise de documentos oficiais pertinentes, como explicado na próxima sessão.

Para melhor compreensão das normas de funcionamento do programa e das recomendações realizadas pelo governo federal aos estados e municípios, foi realizado um levantamento dos todos os atos normativos que se referem diretamente ao PSE e ou a ele são correlatos. Foram levantados também demais documentos oficiais divulgados pelo governo, como manuais, relatórios e material de divulgação.

O levantamento dos atos normativos foi feito inicialmente por busca pelo termo "Programa Saúde na Escola" no Diário Oficial da União. A partir de então, foram adicionados todos os demais atos mencionados na listagem primária obtida. Os outros documentos foram obtidos nas páginas virtuais dos ministérios envolvidos. O Apêndice A traz a listagem completa dos documentos analisados, por ordem cronológica. Todos os textos analisados foram lidos na íntegra e classificados de acordo com as seguintes categorias (Quadro 01): Quadro 1 - Categorias da análise documental

\begin{tabular}{|c|c|}
\hline Categoria & Possíveis classificações \\
\hline Órgão emissor & $\begin{array}{l}\text { Ministério da Saúde (MS) } \\
\text { Ministério da Educação (MEC) } \\
\text { Interministerial (MS e MEC) }\end{array}$ \\
\hline Ano de emissão & 2007 a 2018 \\
\hline Finalidade & $\begin{array}{l}\text { Definição/redefinição de normas } \\
\text { Listagem de municípios (adesão) } \\
\text { Listagem de municípios (repasse } \\
\text { de recursos) } \\
\text { Ações/programas aderentes } \\
\text { Orientações para municípios } \\
\text { Relatório }\end{array}$ \\
\hline
\end{tabular}

Fonte: os autores.

No total foram analisados 51 documentos (Gráfico 1), sendo 38 atos normativos, dentre definições ou redefinições de normas (8), listagem de municípios para recebimento de repasses financeiros (19) e atos relativos a outras ações ou programas aderentes (11). Incluem o conjunto ainda 2 relatórios e 11 documentos de orientação para implementação por parte dos entes federativos, com denominações diversas (guia, manual, passo-a-passo, caderno).

\footnotetext{
${ }^{1}$ Decreto $\mathrm{n}^{\circ} 6.286$, de 5 de dezembro de 2007.

2 Portal do FNS, aba "Consulta de pagamento detalhada", disponível em $<\mathrm{ht}$ tps://consultafns.saude.gov.br/\#/detalhada>.
} 
GRÁFICO 1 - Distribuição dos documentos segundo tipo

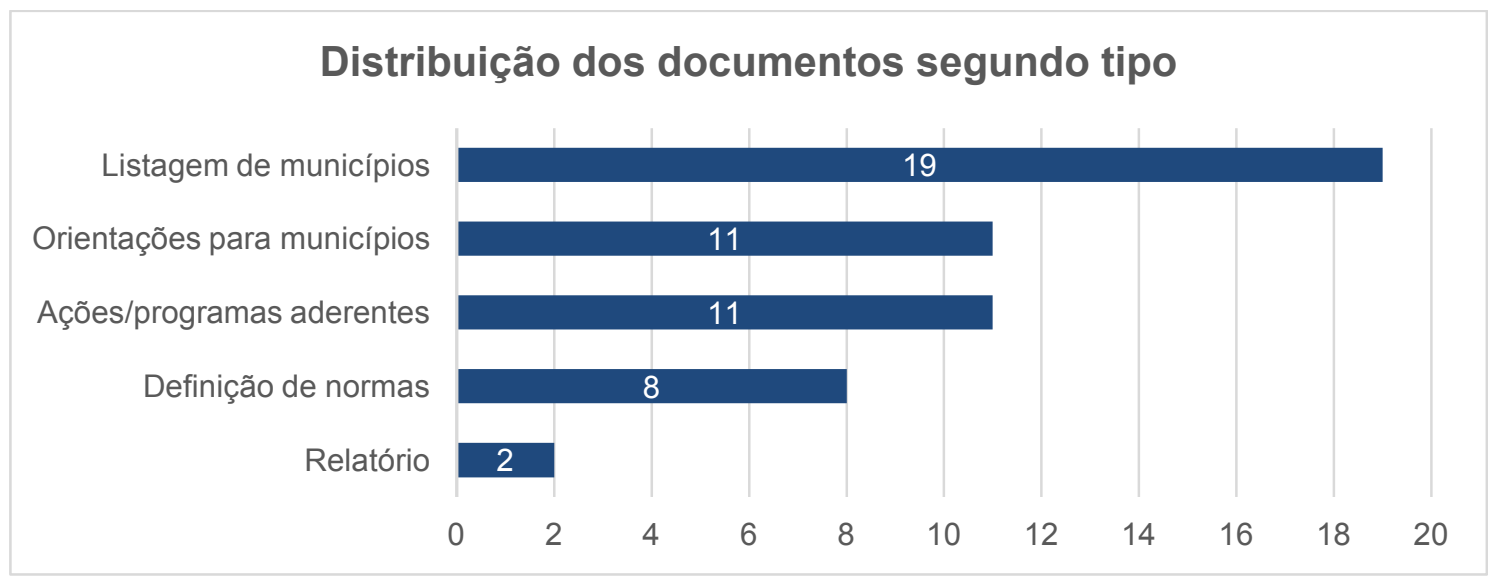

Fonte: dados da pesquisa.

Analisando os anos de publicação dos documentos (GRÁFICO 2), percebe-se uma alta no ano de 2013, quando houve a primeira redefinição das normas do PSE. Nesse ano foram definidas ainda várias questões relativas outros programas e ações aderentes ao PSE, como o evento 'Semana da Saúde na Escola'.

GRÁFICO 2 - Distribuição dos documentos segundo ano de publicação

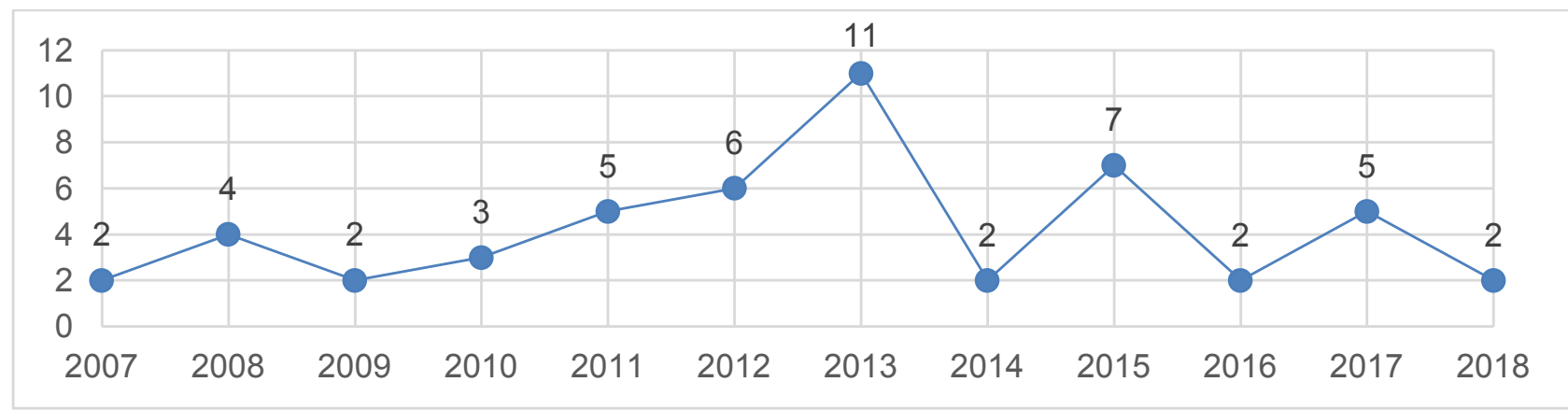

Fonte: dados da pesquisa.

Quanto aos órgãos de publicação, apenas 13 documentos (26\%) são interministeriais (MS e MEC), um número baixo em se tratando de uma política essencialmente intersetorial, desenvolvido em conjunto pelos dois ministérios. O MS responde sozinho pela autoria de $72 \%$ dos documentos. Em 2008 é instituída a Comissão Intersetorial de Educação e Saúde na Escola (CIESE) ${ }^{1}$, responsável pela coordenação do PSE a nível federal.

\section{DESCRIÇÃO DO PROGRAMA SAÚDE NA ESCOLA}

Com a leitura dos documentos foi possível perceber que o programa manteve seus objetivos e diretrizes ao longo do tempo, mas as normas de funcionamento sofreram alterações consideráveis. O Quadro 2 traz a compilação de todas as campanhas, divididas por ano de adesão (como considerado pelo governo federal), mostrando os critérios para definir os municípios aptos, para cálculo e transferência dos recursos.

Apesar de inicialmente pensado para execução anual, na prática a periodicidade de execução, monitoramento e repasses do PSE é irregular. Os órgãos governamentais federais envolvidos falam em campanhas, se baseando no nas datas de publicação das portarias referentes à adesão dos municípios. Por tal lógica, o programa teve início em 2008 e teve no total 10 campanhas, até 2017, quando houve mudanças na sua estrutura, inclusive passando a tratar de ciclos

\footnotetext{
${ }^{1}$ Portaria interministerial no ${ }^{\circ} 675$, de 4 de junho de 2008.
} 
bianuais, com avaliação anual. $\mathrm{O}$ desenho inicial do programa traz uma lista de atividades sugeridas a serem realizadas pelos municípios, divididas da seguinte forma (Ministério da Saúde, 2006):

Componente I - Avaliação das Condições de Saúde das crianças, adolescentes e jovens que estão na escola pública: avaliação clínica, saúde bucal, imunização; avaliação antropométrica, avaliação oftalmológica e psicossocial;

Componente II - Promoção da Saúde e Prevenção: ações de segurança alimentar e promoção da alimentação saudável, promoção das práticas corporais e de atividades físicas, promoção da cultura de paz e prevenção das violências, prevenção ao uso de álcool, tabaco e outras drogas, promoção da saúde ambiental e desenvolvimento sustentável e prevenção às DSTs/AIDS e saúde reprodutiva;

Componente III - Educação permanente e capacitação dos profissionais da educação e da saúde e de jovens.

O primeiro trata do monitoramento da saúde individual dos estudantes, o segundo de atividades educativas, visando conscientização, e o terceiro da inclusão de atividades de formação para os gestores do GTI, profissionais da saúde e da educação envolvidos no PSE e de estudantes para atuarem como multiplicadores. Em alguns documentos de 2009 são mencionados também Componentes IV -Monitoramento e avaliação da saúde dos estudantes e V - Monitoramento e avaliação do PSE, porém eles não aparecem na maioria dos documentos oficiais.

A proposta inicial prevê gestão compartilhada entre as três esferas de governo, responsabilizando a União pela regulamentação e o monitoramento, os municípios pela implementação e os estados pela articulação vertical e horizontal, mobilização e apoio institucional. A participação dos municípios no programa é facultativa. Os critérios de seleção de municípios envolvem o Índice de Desenvolvimento da Educação Básica (IDEB) e a cobertura de atendimento pelas Equipes de Saúde da Família (EqSF), ou a existência de escolas participantes do programa Mais Educação.

QUADRO 2 - Detalhamento dos critérios de adesão e repasse de recursos financeiros

\begin{tabular}{|c|c|c|c|}
\hline $\begin{array}{l}\text { Ano de } \\
\text { adesão } \\
\text { (MEC) }\end{array}$ & Critérios de adesão & Cálculo do valor & Critérios para repasse \\
\hline 2008 & $\begin{array}{l}\text { [(IDEB <2.69 OU } 20 \text { menores de cada estado) e } \\
\text { Cobertura EqSF de } 100 \% \text { ] } \\
\text { OU Possuir escola(s) no programa Mais Educação }\end{array}$ & \multirow{4}{*}{$\begin{array}{l}\text { Equivalente a } 1 \text { mensalidade } \\
\text { das EqSF a mais por cada } \\
\text { EqSF vinculada }\end{array}$} & \multirow{3}{*}{$\begin{array}{l}\text { Parcela única, pela adesão (transferência } \\
\text { feita após a implementação) }\end{array}$} \\
\hline 2009 & $\begin{array}{l}\text { [(IDEB <3.10 OU } 20 \text { menores de cada estado) e } \\
\text { Cobertura EqSF de } 100 \% \text { ] } \\
\text { OU Possuir escola(s) no programa Mais Educação }\end{array}$ & & \\
\hline 2010 & $\begin{array}{l}\text { [(IDEB <4.50 OU } 20 \text { menores de cada estado) e } \\
\text { Cobertura EqSF de } 100 \% \text { ] } \\
\text { OU Possuir escola(s) no programa Mais Educação }\end{array}$ & & \\
\hline $\begin{array}{l}2011 \\
2012\end{array}$ & $\begin{array}{l}\text { [(IDEB <4.50 OU } 20 \text { menores de cada estado) e } \\
\text { Cobertura EqSF de } 100 \% \text { ] } \\
\text { OU Possuir escola(s) no programa Mais Educação }\end{array}$ & & $\begin{array}{l}70 \% \text { iniciais }+30 \% \text { (se atingir } 70 \% \text { das } \\
\text { metas) }\end{array}$ \\
\hline 2012 & $\begin{array}{l}122 \text { municípios considerados prioritários no } \\
\text { Programa Brasil Carinhoso - Programa Brasil } \\
\text { Sem Miséria }\end{array}$ & $\begin{array}{l}\text { A cada } 200 \text { educandos }= \\
\mathrm{R} \$ 1.000,00 \text { (teto: quantidade } \\
\text { de matrículas ativas) }\end{array}$ & $\begin{array}{l}\text { Parcela única, proporcional às metas (se } \\
\text { cumprir mais de } 50 \% \text { ) }\end{array}$ \\
\hline 2013 & \multirow{2}{*}{ Qualquer município } & Até 599 educandos $=\mathrm{R} \$ 3.000$ & $20 \%$ iniciais $+80 \%$ proporcional às metas \\
\hline 2014 & & A cada $+200=+R \$ 1.000$ & (se cumprir mais de $50 \%$ ) \\
\hline $\begin{array}{l}2017 \\
2018\end{array}$ & $\begin{array}{l}\text { Todos os municípios }+ \text { municípios prioritários: } \\
\text { (altas taxas de obesidade infantil) }\end{array}$ & $\begin{array}{l}\text { Até } 600 \text { educandos }=\mathrm{R} \$ \\
5.676,00 \\
\text { A cada }+800=+\mathrm{R} \$ 1.000\end{array}$ & $\begin{array}{l}\text { Parcela única, na adesão. Perde direito a } \\
\text { repasse no segundo ano: } \\
\text { * Se realizar menos de } 2 \text { ações } \\
\text { * Se não realizar a ação } 12 \text { (aedes aegypt) } \\
\text { * Se realizar ações em apenas } 1 \text { escola }\end{array}$ \\
\hline
\end{tabular}

Fonte: dados da pesquisa. 
Cada GTI municipal é responsável por elaborar um plano de ação, a partir de um diagnóstico das questões de saúde mais relevantes na cidade, escolas prioritárias e viabilidade operacional de implementação do programa. É feito então um Termo de Adesão, pelo qual são 'pactuadas' (termo oficial) as EqSF e escolas a serem envolvidas, a quantidades de alunos a serem beneficiados e as ações programadas.

A partir da adesão os municípios devem receber repasses de recursos financeiros para custear as atividades planejadas (MINISTÉRIO DA SAÚDE, 2007). Nas esferas subnacionais os trabalhos devem ser conduzidos por Grupos de Trabalho Intersetoriais (GTI) que incluam, no mínimo, as áreas de saúde e educação, podendo também envolver outros setores interessados, como assistência social e meio ambiente, bem como parceiros externos não pertencentes ao setor público. A execução das ações deve ser realizada conjuntamente por Equipes de Saúde da Família (EqSF) e profissionais da educação (MINISTÉRIO DA SAÚDE, 2007).

O programa prevê a distinção entre escolas prioritárias e não prioritárias, com a regra de que deve haver no mínimo $50 \%$ do primeiro caso no total de pactuadas, que atendam a um dos seguintes perfis: a) creches públicas e conveniadas do município; escolas rurais; escolas com alunos em cumprimento de medidas socioeducativas; escolas que tenham, pelo menos, $50 \%$ de matrículas de alunos pertencentes a famílias beneficiárias do Programa Bolsa Família. Todavia nos relatórios de monitoramento e portarias de repasse não há menção desta distinção.

Observando o Quadro XX, nota-se que os primeiros anos de implementação foram concebidos como uma fase piloto, seguida de ampliação, em três etapas. Para o início do PSE (adesão em 2008) ${ }^{1}$ foram definidos mecanismos de seleção para limitar a quantidade de municípios aptos a participar, baseados nos IDEB municipais para os anos iniciais das escolas públicas e na cobertura da população por EqSF. Foram considerados aptos também municípios que tivessem escolas participantes do programa Mais Educação. A ampliação ocorre nos dois anos seguintes (adesão em 2009 e 2010$)^{2}$, com flexibilização progressiva critérios

\footnotetext{
${ }^{1}$ Portaria MS no 1.861 , de abril de 2008; Portaria MS nº 2.931, de dezembro de 2008; Portaria MS no 3.918 de outubro de 2010.

${ }^{2}$ Portaria MS n 3.146 de dezembro de 2009; Portaria MS nº 1.537 de junho de 2010; Portaria MS n 3.918 de outubro de 2010; Portaria MS/MEC nº
}

iniciais, no intuito de aumentar a quantidade de municípios aptos a aderir ao PSE. Durante esse triênio a transferência de recursos ocorreu com base na quantidade de EqSF vinculadas ao programa e foi feito apenas uma transferência financeira por município, após a execução das ações e registro nos sistemas de monitoramento.

Em 2011 e $2012^{3}$, considera-se encerrada a fase piloto e mantém-se os padrões de seleção de 2010 , adicionando ainda 122 municípios considerados prioritários na ação Brasil Carinhoso, do programa Brasil Sem Miséria. O cálculo do valor do repasse passa a ser feito com base na quantidade de educandos vinculados, em vez de EqSF, como era anteriormente. Os recursos são repassados agora em duas parcelas, uma antes da implementação e outra depois, vinculada ao cumprimento das metas pactuadas, medidas pela quantidade de alunos pactuados.

Cria-se, em março 2012, a Semana Saúde na Escola $^{4}$, que pode ser realizada pelos municípios com participação credenciada no PSE. O evento tem datas e tema anualmente fixados pelo Ministério da Saúde. As ações realizadas durante o evento podem contar como cumprimento de metas do PSE, havendo envio de recursos extra para a realização de atividades específicas. Os critérios para cálculo dos valores a serem repassados são alterados no ano seguinte ${ }^{5}$. O evento passa a ser desvinculado do PSE em junho de $2015^{6}$, podendo ser realizado por qualquer escola interessada, mas ainda com as atividades contando para as metas do programa, para aqueles que dele participam.

Em julho de 2013 tem início a segunda configuração do $\mathrm{PSE}^{7}$, universalizando a possibilidade de adesão, que a partir de então é liberada a qualquer município. $\mathrm{O}$ repasse continua sendo feito em duas parcelas, uma antes da implementação e uma depois, condicionada ao cumprimento das metas. Há alterações no cálculo do valor total a ser repassado, na proporção do valor pago em cada parcela e no percentual mínimo de atingimento das metas para recebimento

\footnotetext{
3.696 de novembro de 2010; Portaria MS/MEC nº 1.911 de agosto de 2011. ${ }^{3}$ Portaria MS/MEC nº 1.910 de agosto de 2011; Portaria MS/GM No 3.014 de dezembro de 2011; Portaria MS n 357 de março de 2012; Portaria MS/GM $\mathrm{N}^{\circ} 524$ de março de 2012; Portaria MS 2.693 de dezembro de 2012; Portaria MS n 297 de fevereiro de 2013.

${ }^{4}$ Portaria MS no 357 de $1^{\circ}$ de março de 2012.

${ }^{5}$ Portaria MS/GM Nº 364 de 08 março de 2013.

${ }^{6}$ Portaria MS no 798 de 17 de junho de 2015.

${ }^{7}$ Portaria MS/MEC no 1.413 de 10 de julho de 2013.
} 
da segunda parte ${ }^{8}$. É o único ano em que o valor final é pago proporcionalmente às metas cumpridas.

Em 2014 mantém-se a configuração de 2013, com adesão no segundo semestre, mas envio dos recursos iniciais apenas em julho de 2015, portanto a maior parte da implementação ocorrendo no final de 2015 e no ano de 2016, não havendo novas adesões nesses anos. O repasse dos recursos finais (para aqueles que cumpriram metas) acontece em dezembro de $2016^{9}$.

Durante o período de implementação, entre 2008 e 2016, os GTI municipais eram responsáveis por registrar a realização das atividades em dois locais, com informações coletadas das equipes de saúde e de educação envolvidas. Para o Componente I, o acompanhamento seria feito pelo Sistema de Informação em Saúde para a Atenção Básica (SISAB), para os demais componentes, no Sistema Integrado de Monitoramento, Execução e Controle (SiMEC).

Percebe-se, no entanto, pelos relatórios emitidos pelo MS e pelas portarias de habilitação de municípios para recebimento de recursos por cumprimento de metas, que o acompanhamento foi feito apenas para as ações de avaliação clínica, registradas no SISAB, por meio do eSUS-AB ${ }^{10}$. Não há manifestações do MEC quanto a controle das informações que deveriam ser monitoradas pelo SiMEC.

Em abril 2017 tem início a terceira configuração do programa ${ }^{11}$. Mantém-se a possibilidade de adesão por qualquer município e o cálculo dos recursos financeiros com base na quantidade de educandos vinculados, mas agora com alteração na metodologia de cálculo e reajuste no valor, que deve ser repassado antes da implementação, nos dois anos previstos como duração desta etapa. A Semana da Saúde continua existindo, mas sem envio de recursos específicos para sua realização. Não há mais a divisão dos "componentes", a lista de ações sugeridas é reformulada e são estabelecidos alguns requisitos para que se tenha direito ao financiamento no segundo ano, incluindo uma força-tarefa direcionada ao mosquito aedes aegypt. São definidos ainda alguns municípios onde o

\footnotetext{
${ }^{8}$ Portaria MS no 2.608 de outubro de 2013; Portaria MS no 1.260 de agosto de 2015.

${ }^{9}$ Portaria MS no 1.337 de setembro de 2015; Portaria MS no 1.067 de julho de 2015; Portaria MS no 2.744 de dezembro de 2016

${ }^{10}$ Software do Ministério da Saúde para alimentação do SISAB, acessível aos municípios.

${ }^{11}$ Portaria MS no 1.055 de 25 de abril de 2017.
}

problema da obesidade é considerado grave, que podem receber recursos extras para atuar em tal frente.

A partir de então o monitoramento das ações passa a ser feito apenas pelo eSUS-AB, não havendo mais registro no SiMEC. Não há definição de quantidade de alunos pactuados ou percentual mínimo a ser cumprido, pois todas as escolas envolvidas no PSE devem ter cobertura total. São criados três indicadores para controle: 1) quantidade de tipos de ações realizadas por escola no conjunto das ações pactuadas, incluindo as adicionadas pelo município, exceto Aedes Aegypti; 2) cobertura das ações de combate ao mosquito Aedes Aegypti nas escolas pactuadas; 3) cobertura do PSE nas escolas pactuadas na adesão. No segundo ano do ciclo poderão ser feitos ajustes na pactuação com a inclusão ou substituição de escolas e atividades. $\mathrm{O}$ "Documento Orientador: Indicadores e Padrões de Avaliação - PSE Ciclo 2017/2018”, publicado pelo $\mathrm{MS}$, traz o seguinte resumo quanto às alterações:

Pela confrontação dos dados contidos nos documentos oficiais analisados, em especial as listagens de habilitação dos municípios para adesão ao PSE e recebimento dos repasses de recursos financeiros, foi possível perceber diversas inconsistências, como: Em todos os anos, envio do valor correspondente à parcela final a municípios sem que eles tenham recebido o repasse inicial, indicativo de que haviam aderido ao programa; Nos primeiros anos, quando havia critérios de adesão e uma listagem preliminar de municípios aptos, casos de localidades que não estavam na habilitação inicial, mas receberam parcela inicial ou final de recursos; Repetição da situação mencionada acima, no caso das Semanas de Saúde, quando houve listagem especificando municípios que já haviam aderido ao programa, portanto estariam aptos a receber recursos extra para a realização do evento, mas não haviam aparecido na habilitação inicial; Municípios que informaram pactuar um número de EqSF maior do que a quantidade autorizada nas portarias que relacionavam tal aspecto e até mesmo maiores do que a quantidade habilitada pelo SUS para aquela localidade; Municípios cuja quantidade de estudantes pactuada é superior à de matriculados; Valores de transferência das parcelas inicial e final não proporcionais, considerando a metodologia de cálculo estabelecida para cada ano. 


\section{CONSIDERAÇÕES FINAIS}

O presente trabalho buscou compreender a trajetória normativa do PSE, após uma década de existência, na qual o programa passou por várias configurações. A análise documental dos atos normativos e publicações oficiais relacionadas ao PSE permitiram tecer um panorama descritivo do programa, com a identificação de nuances inerentes ao seu desenho em cada uma das etapas constatadas.

Foi possível constatar que o programa mantém os aspectos essenciais da proposta inicial, como objetivos, diretrizes e distribuição de responsabilidades entre os entes federativos; mas sofreu alterações consideráveis na estrutura normativa. Foram identificadas as seguintes fases: 1) piloto (de 2008 a 2010), com seleção de municípios aptos a aderirem, repasse de recursos baseado nas EqSF e não vinculados ao desempenho e ampliação progressiva da abrangência, pela flexibilização dos critérios de seleção; 2) expansão (2011 e 2012), mantendo os critérios de seleção de municípios amplos; o cálculo dos repasses passa a ser feito com base na quantidade de alunos vinculados, com parte vinculada ao atingimento de metas; 3 ) universalização (2013 a 2016), estando então disponível para qualquer município; há alterações nos cálculos dos repasses e maior rigorosidade no monitoramento das metas, mas não são seguidos os cronogramas previstos; 4) consolidação (a partir de 2017), quando há uma grande reestruturação nas normas, a partir do que foi observado como prós e contras nos anos anteriores; permanece a possibilidade de adesão por qualquer município; são alterados os valores de repasse, que continuam vinculados ao desempenho; é alterada e flexibilizada a determinação de ações sugeridas aos municípios; assume-se o protagonismo da área da saúde, embora a política continue tendo caráter intersetorial.

Cabe comentar que a periodicidade do programa, inicialmente pensada como anual, na prática foi bastante irregular, sendo alterada para bianual na última reestruturação. Vale lembrar ainda as diversas inconsistências encontradas quando as informações dos documentos são confrontadas, evidenciando falhas no monitoramento do programa, que também foi alterado nessa terceira configuração. Aparentemente, as alterações foram realizadas no intuito de se adequar ao que foi sendo percebido na prática, ao longo das etapas iniciais.
A principal limitação do trabalho é o escopo restrito aos documentos federais como única fonte de informação. A partir disso, são apresentadas as seguintes sugestões para estudos futuros: englobar relatórios locais; ampliar as fontes, coletando dados por questionários ou entrevistas com informantes-chave como gestores, profissionais de saúde e educação, estudantes e familiares; triangular as informações a partir dessa diversificação; realizar análises quantitativas com as informações contidas nas listagens de municípios; buscar sanar os problemas de confiabilidade dos dados a partir da verificação das informações em outras fontes.

\section{REFERÊNCIAS}

AZEVEDO, E.; PELICIONI, M. C. F.; WESTPHAL, M. F. Práticas intersetoriais nas políticas públicas de promoção de saúde. Physis: Revista de Saúde Coletiva, v. 22, p. 1333 1356, 2012.

BUSS, P. M. Health promotion and quality of life. Ciência \& Saúde Coletiva, v. 5, n. 1, 2000, p. 163-77.

CARMO, M. E.; GUIZARDI, F. L. Desafios da intersetorialidade nas políticas públicas de saúde e assistência social: uma revisão do estado da arte. Physis: Revista de Saúde Coletiva, v. 27, dez. 2017,p. 1265-1286.

CASEMIRO, J. P.; FONSECA, A. B. C.; SECCO, F. V. M. Promover saúde na escola: reflexões a partir de uma revisão sobre saúde escolar na América Latina. Ciência \& Saúde Coletiva, v. 19, p. 829-840, 2014.

COUTO, A. N. et al. O ambiente escolar e as ações de promoção da saúde. Cinergis, v. 17, n. 4 Supl.1, p. 378-383, 2016.

CUMMINGS, E. M.; SCHERMERHORN, A. C. A developmental perspective on children as agents in the family. In: KUCZYNSKI, L. (Ed.). . Handbook of dynamics in parent-child relations. California: Sage Publications, 2003. p. 91-108.

DAHLGREN, G.; WHITEHEAD, M. Policies and strategies to promote social equity in health. Stockholm: Institute for Futures Studies, 1991.

DUBOIS, A.; ST-PIERRE, L.; VERAS, M. Revisão de âmbito das definições e modelos conceituais de ações intersetoriais. Ciência \& Saúde Coletiva, v. 20, n. 10, out. 2015, p. 2933-2942.

FARIAS, I. C. V. Análise da Intersetorialidade no Programa Saúde na Escola no Município de Olinda-PE: perspectivas da saúde e da educação. Mestrado em Saúde 
Coletiva-Recife: Universidade Federal de Pernambuco, 2014.

FERREIRA, V. S. C.; SILVA, L. M. V. Intersetorialidade em saúde: um estudo de caso. In: HARTZ, Z. M. A.; SILVA, L. M. V. (Eds.). Avaliação em saúde: dos modelos teóricos à prática na avaliação de programas e sistemas de saúde. EDUFBA / Fiocruz ed. Salvador / Rio de Janeiro: [s.n.].

FIGUEIREDO, M. F.; FIGUEIREDO, A. M. C. Avaliação política e avaliação de políticas: um quadro de referência teórica. São Paulo: Instituto de Estudos Econômicos, Sociais e Políticos de São Paulo, 1986.

FREY, K. Crise do Estado e estilos de gestão municipal. Lua nova, v. 37,1996 , p. $107-138$

GARCIA, L. M. T. et al. Intersetorialidade na saúde no Brasil no início do século XXI: um retrato das experiências. Saúde em Debate, v. 38, n. 103, 2014.

HOLLAND, S. Child and family assessment in social work practice. London: Sage, 2010.

HOWLETT, M.; RAMESH, M.; PERL, A. Política pública: seus ciclos e subsistemas - uma abordagem integral. Tradução Francisco G. Heidemann. Rio de Janeiro: Elsevier, 2013.

INOJOSA, R. M. Intersetorialidade e a configuração de um novo paradigma organizacional. Revista de Administração Pública, v. 32, n. 2, 21 mar. 1998, p. 35-48.

JENKINS, W. I. Policy analysis: A political and organisational perspective. London: M. Robertson, 1978.

KLIKSBERG, B. Falácias e mitos do desenvolvimento social. Tradução Sandra Trabucco Valenzuela. São Paulo: Editora Cortez, 2003.

MARMOT, M. et al. Closing the gap in a generation: health equity through action on the social determinants of health. The Lancet, v. 372, n. 9650, 8 nov. 2008, p. 1661-1669

MINISTÉRIO DA SAÚDE. Política Nacional de Promoção da Saúde. Brasília: Secretaria de Vigilância em Saúde / Secretaria de Atenção à Saúde, 2006.

MINISTÉRIO DA SAÚDE. Decreto no 6.286, de 5 de Dezembro de 2007. Institui o Programa Saúde na Escola - PSE, e dá outras providências., 2007.

MONTEIRO, P. H. N.; BIZZO, N. A saúde na escola: análise dos documentos de referência nos quarenta anos de obrigatoriedade dos programas de saúde, 1971-2011. História, Ciências, Saúde-Manguinhos, v. 22, n. 2, 19 dez. 2014, p. 411-428

MORROW, B. H. Identifying and mapping community vulnerability. Disasters, v. 23, n. 1, 1999. p. 1-18.

NDUMBE-EYOH, S.; MOFFATT, H. Intersectoral action for health equity: a rapid systematic review. BMC Public Health, v. 13, n. 1056, 9 nov. 2013.
OLIVEIRA, L. R. Políticas públicas sociais: avaliação e integração entre assistência social e saúde pública no estado de São Paulo. Doutorado em AdministraçãoRibeirão Preto: Universidade de São Paulo, 2019.

PARSONS, D. W. Public policy: an introduction to the theory and practice of policy analysis. Cheltenham: Edward Elgar Pub, 1995.

PELICONI, M. C. F. Promoção da Saúde e Meio Ambiente: Uma Trajetória Técnica-Política. In: Educação Ambiental e Sustentabilidade. Barueri: Manole, 2005. p. 413-434.

PIRES, V. Economia da educação: para além do capital humano. São Paulo: Cortez, 2005.

POLLITT, C. Joined-up Government: a Survey. Political Studies Review, v. 1, n. 1, 2003, p. 34-49.

POTVIN, L. Intersectoral action for health: more research is needed! International Journal of Public Health, v. 57, n. 1, 1 fev. 2012, p. 5-6.

PSACHAROPOULOS, G.; PATRINOS, H. A. Returns to investment in education: a further update. Education Economics, v. 12, n. 2, ago. 2004, p. 111-134.

SANTOS, J. L. F.; WESTPHAL, M. F. Práticas emergentes de um novo paradigma de saúde: o papel da universidade. Estudos avançados, v. 13, n. 35, 1999, p. 71-88

SECCHI, L. Políticas públicas: conceitos, esquemas de análise, casos práticos. São Paulo: Cencage Learning, 2010. v. 133

SILVA, C. S.; BODSTEIN, R. C. A. Referencial teórico sobre práticas intersetoriais em Promoção da Saúde na Escola. Ciência \& Saúde Coletiva, v. 21, n. 6, jun. 2016, p. $1777-1788$.

SOLAR, O.; IRWIN, A. A conceptual framework for action on the social determinants of health: debates, policy \& practice, case studies.: Social Determinants of Health. Geneva: World Health Organization, 2010.

VIEIRA, M. E. M. Programa Saúde na Escola: a intersetorialidade em movimento. Mestrado em Processos de Desenvolvimento Humano e Saúde-Brasília: Universidade de Brasília, 2013.

WOOD, L. et al. "Through the Kids... We Connected With Our Community" Children as Catalysts of Social Capital. Environment and Behavior, v. 45, n. 3, 2013, p. 344-368.

WU, X. et al. Guia de políticas públicas: gerenciando processos. Tradução Ricardo A. Souza. Brasília: ENAP, 2014. 